\title{
When the heart rules the head: ischaemic stroke and intracerebral haemorrhage complicating infective endocarditis
}

Estabrak Jiad MBBS ${ }^{1 *}$, Sumanjit K Gill MRCP ${ }^{1,3 *}$, Maria Krutikov MB ChB ${ }^{1 *}$, David Turner MRCP $^{1}$, Michael H Parkinson MRCP ${ }^{1}$, Carmel Curtis FRCPath ${ }^{2}$, David J Werring FRCP $\mathrm{PhD}^{1,3}$

Affiliations:

1 Department of Neurology, National Hospital for Neurology \& Neurosurgery, Queen Square, London, WC1N 3BG, UK

2 Department of Microbiology, University College Hospital, 60 Whitfield St, London, W1T 4EU, UK

3 Stroke Research Centre, Department of Brain Repair \& Rehabilitation, UCL Institute of Neurology, Queen Square, London, WC1N 3BG, UK

*These authors contributed equally

Correspondence to: David J Werring, Professor of Clinical Neurology, Stroke Research Centre, UCL Institute of Neurology, Box 6, National Hospital for Neurology and Neurosurgery, Queen Square, London, WC1N 3BG, UK

Email: d.werring@ucl.ac.uk

Tel: +44 (0) 2031087493

Paper type: Review

Words: 4826 


\begin{abstract}
Sir William Osler meticulously described the clinical manifestations of infective endocarditis in 1885, concluding that: "few diseases present greater difficulties in the way of diagnosis.... which in many cases are practically insurmountable." Even with modern investigation techniques, diagnosing infective endocarditis can be hugely challenging, yet is critically important in patients presenting with stroke (both cerebral infarction and intracranial haemorrhage), its commonest neurological complication. In ischaemic stroke, intravenous thrombolysis carries an unacceptably high risk of intracranial haemorrhage, while in intracerebral haemorrhage, mycotic aneurysms require urgent treatment to avoid re-bleeding; and in all cases, prompt treatment with antibiotics and valve surgery may be life-saving. Here we describe typical presentations of ischaemic stroke and intracerebral haemorrhage caused by infective endocarditis. We review the diagnostic challenges, the importance of rapid diagnosis, treatment options, and controversies.
\end{abstract}

\title{
CLINICAL CASE REPORTS
}

\section{Case Report 1}

A 60-year old man presented to the hyperacute stroke unit with acute speech problems and right-sided hemiparesis. He had a background of diabetes and hypertension. Four months previously he had been admitted with a left middle cerebral artery ischaemic stroke due to a cortical branch occlusion, but no cause had been identified: the extracranial and intracranial arteries were normal and there was no abnormality on a transthoracic echocardiogram or cardiac rhythm monitoring. After full investigation, the stroke was considered to be due to proximal embolism of undetermined source, probably cardiac. His CRP was elevated on admission $95 \mathrm{mg} / \mathrm{L}$ but dropped within the first few days to $33 \mathrm{mg} / \mathrm{L}$ whereupon it seemed to stabilise but never dropped lower than this. After discharge, he reported muscle pain, weight loss and fatigue, for which investigations showed persistently raised inflammatory markers (CRP 61mg/L, ESR 79mm/hr), negative autoimmune screen and low complement C4. A repeat transthoracic echocardiogram and CT-PET of the whole body were normal. He was treated with prednisolone for a presumed diagnosis of polymyalgia rheumatica, with two doses of intravenous steroid given for ongoing muscle pains the week before admission with another stroke. On this second admission, he presented with sudden word-finding difficulties and mild 
right arm weakness. CT imaging showed an acute left posterior cerebral artery territory infarct in the temporo-occipital region and an established left postero-lateral temporal lobe infarct in the middle cerebral artery territory (Figure 1, panel A). He was not within the time window for intravenous thrombolysis. The following day he had a fever with a temperature of $38.4^{\circ} \mathrm{C}$; examination revealed right basal chest crackles, a loud systolic murmur and finger splinter haemorrhages. Blood tests showed CRP $64 \mathrm{mg} / \mathrm{L}$, ESR $40 \mathrm{~mm} / \mathrm{hr}$, and white cell count $8 \times 10^{9} /$ L. Blood cultures grew Abiotrophia defectiva, a nutritionally variant streptococcus constituent of normal oral flora. He was commenced on four hourly high dose intravenous amoxicillin. An urgent transthoracic echocardiogram showed vegetations on the aortic and mitral valves, with moderate to severe aortic and mitral regurgitation but good left ventricular function. Urgent mechanical mitral and aortic valve replacement was performed. He was subsequently anticoagulated with warfarin, and transferred for further neurorehabilitation.

\section{Case Report 2}

A 37-year old man presented to the hyperacute stroke unit with sudden onset left-sided weakness and sensory disturbance. He had no significant past medical history, except that over the past three months he had been feeling non-specifically unwell with intermittent fevers and night sweats. He had attributed these symptoms to a viral infection, which was prevalent in staff and pupils in the school in which he worked as a teacher. GP records indicated that a blood test two months previously had shown an elevated CRP of $96 \mathrm{mg} / \mathrm{L}$ and deranged liver function tests, but examination had revealed no explanation for these findings. When admitted to the stroke unit he had left hemiparesis and sensory loss; a systolic murmur was audible on examination. Admission blood tests showed raised CRP at $66 \mathrm{mg} / \mathrm{L}$, with a normal white cell count. CT head showed an intracerebral haemorrhage in the right frontal lobe (Figure 1, panel B). Subsequent cerebral digital subtraction angiography revealed a $4 \mathrm{~mm}$ mycotic aneurysm (Figure 1, panel C), which was successfully treated by endovascular glue embolisation. An urgent transthoracic echocardiogram showed an $11 \mathrm{~mm}$ vegetation on the posterior leaflet of the mitral valve (Figure 1, panel D). Blood cultures grew Streptococcus sanguinis, a member of the viridans streptococci group, which is present in dental plaque. He was treated with intravenous antibiotics for 6 weeks, and subsequently had a mitral valve repair.

\section{DISCUSSION}


Cerebral complications occur in up to $55 \%$ of patients with infective endocarditis, often before the diagnosis of infective endocarditis is made, and include stroke, brain abscess and meningoencephalitis. ${ }^{1-3}$. In-hospital case fatality of infective endocarditis is about $20 \%,{ }^{1}$ and is even higher with neurological involvement. Stroke is the commonest neurological complication, and occurs in about $35 \%$ of cases of infective endocarditis; a further $30 \%$ have "silent" cerebrovascular lesions (iscahemic or hemorrhagic) detectable on MRI. ${ }^{2}$ Both symptomatic ischaemic stroke and intracerebral haemorrhage occur, the latter sometimes associated with a demonstrable mycotic aneurysm. Mortality from stroke due to infective endocarditis seems to be higher in patients with prosthetic heart valves, intracerebral hemorrhage, Staphylococcus aureus infection, advanced age, cardiac complications, emergency heart surgery, and septic shock.

\section{Diagnosis of endocarditis in patients with stroke}

Infective endocarditis has a highly variable clinical presentation, which may be acute and rapidly progressive, or may have a longer and non-specific prodrome. An acute presentation of infective endocarditis with fevers and congestive heart failure is more easily recognized than a more insidious sub-acute presentation.

The non-specific preceding systemic symptoms in both of our cases - initially attributed to alternative diagnoses - are key to alerting clinicians to the possibility of subacute infective endocarditis as a cause of an acute stroke. It is particularly important that clinicians specifically ask about preceding systemic prodromal symptoms when faced with any unexplained stroke (for example a younger patient with no known vascular risk factors, as in our second case). These symptoms are often non-specific; fevers and rigors/chills are most common, but weight loss, poor appetite, night sweats, headache, general malaise, cough, myalgia and joint pains can all occur. Infective endocarditis should be suspected in any patient with unexplained stroke who has fever (especially with bacteraemia), cardiac risk factors (previous infective endocarditis, valvular heart disease including congenital heart disease, or a prosthetic valve), or non-cardiac risk factors (intravenous drug use, immunosuppression, or a recent dental or surgical procedure). The combination of unexplained symptomatic or asymptomatic ischaemic and haemorrhagic cerebral lesions together (especially if multiple) should also raise suspicion of endocarditis. Cerebral microbleeds on blood-sensitive MRI are seen in about $60 \%$ of patients 
with infective endocarditis, and if numerous (especially in a younger patient) can be a useful clue (Figure 2$)^{4}$.

The diagnosis of infective endocarditis is typically based on a combination of features from amongst the history, examination and investigation results, rather than on a single definitive test. Cardiac auscultation on examination, observation for peripheral stigmata of infective endocarditis, temperature measurement and checking inflammatory markers are also essential in the acute assessment of all patients with acute stroke. Blood cultures and echocardiography are particularly important. Table 1 provides a list of the frequency of clinical and laboratory findings relevant to diagnosing infective endocarditis from a large multicenter study including patients diagnosed with definite infective endocarditis ${ }^{5}$.

The diagnosis may be straightforward if classical and specific signs of endocarditis are present: Janeway lesions (non-tender erythematous macules on the palms and soles); Osler nodes (tender, subcutaneous violaceous nodules on the fingers, toes, thenar or hypothenar eminences) and Roth spots (haemorrhagic, oedematous lesions of the retina with pale centres). Janeway lesions are presumed embolic phenomena, whereas Osler nodes and Roth spots are thought to be immunological in origin. These classical findings are highly specific, but not very common (Table 1). Splinter haemorrhages are not so specific, especially if only one or two are found. Furthermore, the peripheral signs due to systemic embolism can be absent in solely right heart infection, which includes intravenous drug users or those with very acute infection. Although fever is nearly always present in infective endocarditis (in one large study over $95 \%$ of patients had a fever $>38$ degrees $^{5}$ ), in acute stroke, fever is often secondary to a complication, for example due to pneumonia, urinary tract infection (UTI), or sepsis, so fever in isolation is not a useful pointer to the possibility of infective endocarditis. Nearly all patients with left heart infective endocarditis have a heart murmur on auscultation, so absence of this sign should lead to questioning the diagnosis; however, murmurs are not so common in right heart infective endocarditis.

Blood inflammatory markers in infective endocarditis are highly sensitive but not very specific, partly because infections often complicate acute stroke; they include elevated erythrocyte sedimentation rate or C-reactive protein. Since infectious endocarditis is one of the few conditions causing a highly elevated ESR, a level of $>100 \mathrm{~mm} / \mathrm{hr}$ in a patient with acute stroke without an alternative explanation should raise diagnostic suspicion. Positive rheumatoid factor is an immunological phenomenon present in about $5 \%$ of cases. Hyperglobulinaemia, 
cryoglobulinaemia, circulating immune complexes, low complement, and false-positive serologic tests for syphilis can also occur. Microscopic haematuria and proteinuria may be present; red blood cell casts in the urine, suggesting glomerulonephritis, may be particularly helpful, and this finding is a minor diagnostic criterion for infective endocarditis. At least three sets of blood cultures should be taken from separate venepuncture sites before starting antibiotics, which will capture $96 \%$ to $98 \%$ of cases of bacteraemia. ${ }^{6}$ Antibiotics should not be delayed if the diagnosis is suspected and the patient is clinically unstable. Electrocardiography may demonstrate new or evolving conduction disease (first-degree atrioventricular block, bundle branch block, or complete heart block), reflecting paravalvular or myocardial extension of infection. MRI of the brain can be helpful in the diagnosis of stroke due to endocarditis: as well as showing the symptomatic ischaemic or haemorrhagic lesion, asymptomatic abnormalities are very frequently detected. In one study of 130 patients, 106 (82\% [95\% CI, $75 \%$ to $89 \%$ ]) patients had clinically silent lesions; these were ischemic lesions in 68 , microbleeds in 74 , and silent aneurysms in $10 .^{7}$

The Duke diagnostic criteria have been validated in pathologically proven infective endocarditis, with sensitivity $>80 \%^{8}$ and almost $100 \%$ specificity in patients with acute unexplained fever ${ }^{9}$. The modified Duke criteria give major and minor criteria required for the diagnosis of infective endocarditis (Table 2) ${ }^{10}$ : Definite infective endocarditis is diagnosed by: two major clinical criteria; one major and three minor clinical criteria; or five minor clinical criteria. However, these criteria should always be combined with clinical judgement of the pretest probability. The diagnostic accuracy may be lower in suspected prosthetic valve infective endocarditis, right-sided infective endocarditis, cardiac device infection, and has not been evaluated specifically in patients with acute stroke ${ }^{11}$. Thus, the diagnosis of infective endocarditis in a patient with stroke should not be based entirely on fulfillment of these criteria.

A positive blood culture for an organism compatible with infective endocarditis in a stroke patient should always lead the clinician to review the history and seek out the possible diagnosis of infective endocarditis. There is a high yield from a first blood culture: $96 \%$ in streptococcal infective endocarditis and $82 \%$ of cases caused by other organisms in one series of 206 cases. $^{12}$ In those who are culture negative, fastidious organisms (for example Coxiella, and Bartonella) should be considered, and the appropriate serologies sent for confirmation ${ }^{12}$. The HACEK group (Haemophilus species, Actinobacillus actinomycetemcomitans, Cardiobacterium hominis, Eikenella corrodens and Kingella kingae) are fastidious Gram negative organisms which will be cultured by the laboratory if the appropriate clinical details are provided. 
Echocardiography (transthoracic and, if necessary, transoesophageal) should be urgently undertaken in all patients with stroke if infective endocarditis is suspected, as prompt diagnosis allows life-saving treatment, including antibiotics and valve replacement. Transoesophageal echocardiography is more sensitive for diagnosis: in one series, trans-thoracic echocardiography (TTE) only identified vegetations in $32 \%$ of cases, while trans-oesophageal echocardiography did so in $19 \%$ of those with a negative TTE and in $21 \%$ of those with an indeterminate TTE result. ${ }^{13}$ Other echocardiographic findings include abscess and moderate or severe new valvular regurgitation. Vegetation length of longer than $10 \mathrm{~mm}$ has been reported to be predictive of embolic events, while $>15 \mathrm{~mm}$ predicted higher mortality. ${ }^{14}$

\section{Treatment of stroke associated with infective endocarditis}

\section{General treatment}

Optimum management of infective endocarditis always requires input from multiple clinical teams; in stroke patients this might need to include neurologists or stroke physicians, cardiologists and cardiothoracic surgeons, neuroradiologists, neurosurgeons, anaesthetists, and microbiology and infectious disease specialists. Antibiotics are the mainstay of treatment and appear to reduce the risk of stroke from $0.5 \%$ per day to $0.3 \%$ per day ${ }^{15}$. Suggested antibiotic regimens for native and prosthetic valve endocarditis usually include combination intravenous therapy (e.g. amoxicillin and gentamicin for native valve infective endocarditis) but local microbiological expertise is essential. Cardiac valve surgery is needed in about $40-50 \%$ of patients with infective endocarditis ${ }^{16}$ for three main indications: heart failure due to valve dysfunction, uncontrolled infection, and prevention of embolism. The aims of surgery are to eradicate infection and normalize heart anatomy. Surgery may involve valve repair or bioprosthetic or mechanical replacement; specific evidence for the best approach or optimum timing is not available. In practice, because of concerns about the risks of surgery, anaesthesia and cardiopulmonary bypass, valve surgery is traditionally delayed until patients with infective endocarditis-related stroke are neurologically stable, typically for about two weeks. However, recent data suggests that early surgery might be safe in infective endocarditis ${ }^{17}{ }^{18}$. A randomized study of early vs. standard timing of surgery found that death from any cause, embolic events, or recurrence of infective endocarditis at 6 months was 3\% in the early-surgery group and $28 \%$ in the conventional treatment group (hazard ratio, 0.08; $95 \% \mathrm{CI}, 0.01$ to 0.65 ; $\mathrm{P}=0.02) .{ }^{17}$ Current guidelines therefore suggest that after ischaemic stroke, surgery indicated 
for heart failure, uncontrolled infection, abscess, or persistent high embolic risk should be considered early (ie <14 days), in the absence of severely reduced conscious level or haemorrhage on cranial CT or MRI. ${ }^{11}$ In patients with intracranial haemorrhage, neurological prognosis is worse, so it is suggested that surgery should generally be postponed for at least a month. Cardiac surgery typically requires anticoagulation, so particular caution is needed to ensure that intracranial haemorrhage risk is reduced to an acceptable level (e.g. by securing ruptured mycotic aneurysms). In practice, timing is decided by multidisciplinary discussion between the stroke, anaesthetics, cardiology and cardiac surgery teams, and is generally delayed until the patient is neurologically stable.

\section{Ischaemic stroke}

Ischaemic stroke is three times more likely than intracranial haemorrhage in infective endocarditis ${ }^{19}$ and is attributed to the embolization of infected valve material to the brain. The risk of neurologic complications has been suggested to be higher in patients with Staphylococcus aureus infective endocarditis, and with multiple valve involvement. The risk of stroke might be higher with mitral than aortic valve involvement. ${ }^{12,20}$

Intravenous thrombolysis is the standard acute treatment for ischaemic stroke patients presenting within 4.5 hours, but probably carries more hazard than benefit in ischaemic stroke due to infective endocarditis. Intracerebral bleeding in infective endocarditis could occur from either haemorrhagic transformation of an infarct, rupture of an aneurysm, or from a friable inflamed blood vessels due to associated arteritis. The risk of post-thrombolysis intracranial haemorrhage has been reported to be higher in infective endocarditis related ischaemic stroke than in other patients (in one sample of 222 patients the rate of post-thrombolysis intracerebral haemorrhage in infective endocarditis-related ischaemic stroke was $20 \%$, compared to $6.5 \%$ in patients with ischaemic stroke unrelated to infective endocarditis). ${ }^{21}$ Moreover, there was a significantly lower rate of favourable outcome in the infective endocarditis group (10\% versus $37 \% ; \mathrm{P}=0.01$ ). Thus, it is likely that this large absolute increase in intracerebral haemorhage risk outweighs the absolute benefit of treatment in most cases of ischaemic stroke associated with infectious endocarditis. However, only a randomized trial could definitively prove that there is no net benefit from alteplase in stroke due infective endocarditis. Given the rarity of endocarditis related ischaemic stroke, such a trial is not feasible, so clinicians must make an informed judgement based on the observational evidence. In our practice, we try to avoid 
systemic thrombolysis in ischaemic stroke due to infective endocarditis. An alternative treatment for some patients might be endovascular clot retrieval treatment (thrombectomy), which has recently been shown to be dramatically effective in anterior circulation large vessel occlusion; ${ }^{22}$ this option is a theoretically attractive option in ischaemic stroke due to infective endocarditis, but has not been evaluated in clinical trials or observational studies in this specific setting. Furthermore, thrombectomy might have higher risks in infective endocarditis than in the trial populations studied, and cannot currently be routinely offered in all stroke centres.

A key clinical challenge is in recognizing the possibility of infectious endocarditis in the very short time window available for effective intravenous thrombolysis in acute ischaemic stroke: "red flags" to alert clinicians to the possibility include: young age with no risk factors; multiple ischaemic or haemorrhagic brain lesions; known cardiac valve abnormality; intravenous drug use; immunosuppression; preceding malaise, myalgia or weight loss; cardiac murmur with fever; clinical stigmata of embolism or immune complex formation; or extremely raised inflammatory markers (e.g. ESR >100mm / hour).

Anticoagulation and antiplatelet treatments are also potentially hazardous in infective endocarditis, with a substantial risk of intracerebral haemorrhage. Cerebral microbleeds (CMBs) are common in infective endocarditis (Figure 2), and were described in $57 \%$ of patients in one case series of 60 patients; usually lobar, with an average of about 8 microbleeds per patient ${ }^{4}$; they are probably secondary to inflammation and leakage of the microvasculature ${ }^{4}$, which might contribute to the high risk of intracerebral haemorrhage in infective endocarditis, even in the absence of anticoagulation. Intracerebral haemorrhage is especially common with Staphylococcus aureus infection, which can be considered when making antithrombotic decisions. Unfortunately, data to guide the use of anticoagulants and antiplatelet therapies in infective endocarditis are extremely limited. This decision thus presents a dilemma for the clinician who needs to balance the risk of thombo-embolization (which might be reduced by anticoagulation) with the risk of intracranial haemorrhage (which might be aggravated). In general, there is no evidence to support the use of anticoagulants or antiplatelet drugs in acute stroke due to infective endocarditis so these should be avoided in the acute phase in the absence of any compelling indication; intra-cardiac thrombus, atrial fibrillation or venous thromboembolism might be indications for early anticoagulation. The specific question of whether to continue anticoagulants in patients with prosthetic valve endocarditis remains unsettled, and no general recommendation can be made; the decision needs to individualized, taking account of all available clinical, laboratory and imaging 
information. Following infective endocarditis-associated ischaemic stroke with a strong indication for anticoagulation it is generally reasonable to delay this by 2 weeks, as is conventional for other patients with cardioembolic stroke. An urgent priority in infective endocarditis patients at risk of intracerebral haemorrhage is cardiological assessment of valve function, and cerebral angiography to look for mycotic aneurysms as these may be amenable to intervention, rather than early anticoagulation; this should allow anticoagulation and other acute decisions to be based on an informed estimate of the competing risks. In particular this information is vital to allow planning of valve surgery, if required.

\section{Intracerebral haemorrhage}

Intracerebral haemorrhage occurs in approximately 5\% of patients with infective endocarditis and is thought to be due to haemorrhagic transformation of infarcted brain, septic arteritis and vessel wall rupture, or rupture of a mycotic aneurysm ${ }^{23}$. Mycotic aneurysms in infective endocarditis are most frequently found in distal branches of the middle cerebral artery territory. Patients with Staphylococcus aureus bacteraemia are more likely to develop intracerebral haemorrhage in the first 48 hours after hospital admission. The mortality from ruptured mycotic aneurysms has been reported to be as high as $80 \%$. The rebleeding risk of ruptured mycotic aneurysms is likely to be high, and all should therefore be considered for endovascular or surgical treatment. Clipping may be technically difficult due to an often poorly defined aneurysmal neck and vascular fragility Endovascular therapy (coiling or acrylic glue) may be more appropriate, especially in patients who are unfit for surgery due to cardiac disease. Indeed, surgical treatment of a mycotic aneurysm is a viable option in very few patients - one series of 179 patients with infective endocarditis-related intracerebral haemorrhage found only $2.2 \%$ of patients who could be surgically treated. ${ }^{23}$ The risk of bleeding from unruptured mycotic aneurysms is unknown, and patients are not routinely offered treatment for this reason.

In conclusion, infective endocarditis is a diagnostic and management challenge in the context of acute ischaemic stroke and intracerebral haemorrhage. The diagnosis of infective endocarditis has important implications for management, which remains challenging due to a lack of prospective controlled studies. We emphasize the importance of a multidisciplinary collaborative approach to give the best outcomes in this devastating disease.

\section{Key Learning points}


- Diagnosing infective endocarditis in patients with stroke is challenging but important: in ischaemic stroke due to infective endocarditis, systemic thrombolysis carries a high bleeding risk and is probably best avoided; endovascular treatment is an alternative for large vessel anterior occlusions, though not tested in this specific setting

- Non-specific prodromal systemic symptoms are key to alerting clinicians to the possibility of infective endocarditis in a patient with stroke

- Antithrombotic drugs should generally be avoided in acute stroke due to infective endocarditis because of the high risk of intracerebral haemorrhage

- Ruptured mycotic aneurysms have a poor prognosis if left untreated, and require urgent surgical or endovascular treatment

- In any stroke patient with infective endocarditis antibiotics and valve surgery may be lifesaving; current evidence favours early surgery ( $<14$ days) in those with ischaemic stroke 


\section{Figure 1}

(A) Axial CT head of case 1, 6 days after the second admission showing a left occipital infarct (arrowed). (B) Initial axial CT head of case 2 showing right hemisphere frontal intraparenchymal haemorrhage (arrowed) with contrast pooling outlining the aneurysmal sac. (C) Intra-arterial digital subtraction cerebral angiogram of case 2 showing mycotic aneurysm (arrowed). (D) Echocardiogram of case 2 showing a vegetation (arrowed) on the posterior mitral valve leaflet.

\section{Figure 2}

(A) and (B) Axial T2*-weighted gradient-echo MRI showing cerebral microbleeds both supratentorially and infratentorially (white arrows) and a larger hypointense lesion (white arrowhead, left panel), which on intra-arterial digital subtraction angiography was found to be a mycotic aneurysm, subsequently treated with glue embolization. 
Table 1. Clinical and laboratory findings and their prevalence in 2781 patients fulfilling the Duke criteria for infective endocarditis (modified from reference 4)

\begin{tabular}{|l|c|}
\hline Finding & (\%) of Patients \\
\hline Fever of more than $38^{\circ} \mathrm{C}$ & 96 \\
\hline Splinter hemorrhages & 8 \\
\hline Osler nodes & 3 \\
\hline Janeway lesions & 2 \\
\hline Roth spots & 17 \\
\hline Vascular embolic event & 5 \\
\hline Conjunctival hemorrhage & 11 \\
\hline Splenomegaly & 48 \\
\hline New murmur & 20 \\
\hline Worsening of old murmur & 61 \\
\hline Elevated ESR & 26 \\
\hline Elevated C-reactive protein level & \\
\hline Hemated rheumatoid factor & \\
\hline
\end{tabular}


Table 2: Modified Duke criteria for diagnosis of infective endocarditis (from Li et al):

The diagnosis of infective endocarditis is definite with: one pathological criterion; two major criteria; one major and three minor criteria; or five minor criteria

\section{Pathological criteria}

Microorganisms on histology or culture of a vegetation or intracardiac abscess

Vegetation or intracardiac abscess showing active endocarditis on histology

\section{Major clinical criteria}

1) Blood cultures positive for infective endocarditis

Typical microorganisms consistent with IE from two separate blood cultures: - Staphylococcus aureus, viridans streptococci, Streptococcus bovis, HACEK (Haemophilus, Aggregatibacter, Cardiobacterium, Eikenella corrodens, Kingella) group, or communityacquired enterococci, in the absence of a primary focus

or

Microorganisms consistent with infective endocarditis from persistently positive blood cultures:

- At least two positive blood cultures from blood samples drawn $>12 \mathrm{~h}$ apart, or

- All of three, or most of $\geq 4$ separate cultures (with first and last sample $>1 \mathrm{~h}$ apart) or

- Single positive culture for Coxiella burnetii, or phase $1 \mathrm{IgG}$ antibody titre >1:800

2) Evidence of endocardial involvement

Echocardiography positive for infective endocarditis

- Defined by presence of a vegetation, abscess, or new partial dehiscence of prosthetic valve New valvular regurgitation (note: increase or change in pre-existing murmur is not sufficient)

\section{Minor clinical criteria}

1) Predisposition: predisposing heart condition, intravenous drug use

2) Fever: temperature $>38^{\circ} \mathrm{C}$

3) Vascular phenomena: major arterial emboli, septic pulmonary infarcts, mycotic aneurysm, intracranial haemorrhages, conjunctival haemorrhages, Janeway lesions

4) Immunological phenomena: glomerulonephritis, Osler nodes, Roth spots, rheumatoid factor

5) Microbiological evidence: positive blood culture that does not meet a major criterion or serological evidence of active infection with organism consistent with infective endocarditis 


\section{Contributions}

All authors cared for the patients. DJW had the idea for the paper. EJ, SG, MK, DT, MHP and DJW wrote the manuscript. Written consent to publication was obtained from both patients. 


\section{References}

1. Hoen B, Duval X. Clinical practice. Infective endocarditis. The New England journal of medicine 2013;368(15):1425-33. doi: 10.1056/NEJMcp1206782

2. Snygg-Martin U, Gustafsson L, Rosengren L, et al. Cerebrovascular complications in patients with left-sided infective endocarditis are common: a prospective study using magnetic resonance imaging and neurochemical brain damage markers. Clinical infectious diseases : an official publication of the Infectious Diseases Society of America 2008;47(1):23-30. doi: 10.1086/588663

3. Sonneville R, Mirabel M, Hajage D, et al. Neurologic complications and outcomes of infective endocarditis in critically ill patients: the ENDOcardite en REAnimation prospective multicenter study. Critical care medicine 2011;39(6):1474-81. doi: 10.1097/CCM.0b013e3182120b41

4. Klein I, lung B, Labreuche J, et al. Cerebral microbleeds are frequent in infective endocarditis: a casecontrol study. Stroke; a journal of cerebral circulation 2009;40(11):3461-5. doi: 10.1161/STROKEAHA.109.562546

5. Murdoch DR, Corey GR, Hoen B, et al. Clinical presentation, etiology, and outcome of infective endocarditis in the 21st century: the International Collaboration on Endocarditis-Prospective Cohort Study. Archives of internal medicine 2009;169(5):463-73. doi: 10.1001/archinternmed.2008.603

6. Cahill TJ, Prendergast BD. Infective endocarditis. Lancet 2016;387(10021):882-93. doi: 10.1016/S0140-6736(15)00067-7

7. Thuny F, Gaubert JY, Jacquier A, et al. Imaging investigations in infective endocarditis: current approach and perspectives. Archives of cardiovascular diseases 2013;106(1):52-62. doi: 10.1016/j.acvd.2012.09.004

8. Habib G, Derumeaux G, Avierinos JF, et al. Value and limitations of the Duke criteria for the diagnosis of infective endocarditis. Journal of the American College of Cardiology 1999;33(7):2023-9.

9. Hoen B, Beguinot I, Rabaud C, et al. The Duke criteria for diagnosing infective endocarditis are specific: analysis of 100 patients with acute fever or fever of unknown origin. Clin Infect Dis 1996;23(2):298-302.

10. Li JS, Sexton DJ, Mick N, et al. Proposed modifications to the Duke criteria for the diagnosis of infective endocarditis. Clinical infectious diseases : an official publication of the Infectious Diseases Society of America 2000;30(4):633-8. doi: 10.1086/313753

11. Habib G, Lancellotti P, Antunes MJ, et al. 2015 ESC Guidelines for the management of infective endocarditis: The Task Force for the Management of Infective Endocarditis of the European Society of Cardiology (ESC). Endorsed by: European Association for Cardio-Thoracic Surgery (EACTS), the European Association of Nuclear Medicine (EANM). European heart journal 2015;36(44):3075-128. doi: 10.1093/eurheartj/ehv319

12. Werner AS, Cobbs CG, Kaye D, et al. Studies on the bacteremia of bacterial endocarditis. Jama 1967;202(3):199-203.

13. Fowler VG, Jr., Li J, Corey GR, et al. Role of echocardiography in evaluation of patients with Staphylococcus aureus bacteremia: experience in 103 patients. Journal of the American College of Cardiology 1997;30(4):1072-8.

14. Thuny F, Di Salvo G, Belliard O, et al. Risk of embolism and death in infective endocarditis: prognostic value of echocardiography: a prospective multicenter study. Circulation 2005;112(1):69-75. doi: 10.1161/CIRCULATIONAHA.104.493155

15. Hart RG, Foster JW, Luther MF, et al. Stroke in infective endocarditis. Stroke; a journal of cerebral circulation 1990;21(5):695-700.

16. Prendergast BD, Tornos P. Surgery for infective endocarditis: who and when? Circulation 2010;121(9):1141-52. doi: 10.1161/CIRCULATIONAHA.108.773598 
17. Kang DH, Kim YJ, Kim SH, et al. Early surgery versus conventional treatment for infective endocarditis. The New England journal of medicine 2012;366(26):2466-73. doi: 10.1056/NEJMoa1112843

18. Ruttmann E, Willeit J, Ulmer $\mathrm{H}$, et al. Neurological outcome of septic cardioembolic stroke after infective endocarditis. Stroke; a journal of cerebral circulation 2006;37(8):2094-9. doi: 10.1161/01.STR.0000229894.28591.3f

19. Heiro M, Nikoskelainen J, Engblom E, et al. Neurologic manifestations of infective endocarditis: a 17-year experience in a teaching hospital in Finland. Archives of internal medicine 2000;160(18):2781-7.

20. Anderson DJ, Goldstein LB, Wilkinson WE, et al. Stroke location, characterization, severity, and outcome in mitral vs aortic valve endocarditis. Neurology 2003;61(10):1341-6.

21. Asaithambi G, Adil MM, Qureshi Al. Thrombolysis for ischemic stroke associated with infective endocarditis: results from the nationwide inpatient sample. Stroke; a journal of cerebral circulation 2013;44(10):2917-9. doi: 10.1161/STROKEAHA.113.001602

22. Goyal M, Menon BK, van Zwam WH, et al. Endovascular thrombectomy after large-vessel ischaemic stroke: a meta-analysis of individual patient data from five randomised trials. Lancet 2016;387(10029):1723-31. doi: 10.1016/S0140-6736(16)00163-X

23. Hart RG, Kagan-Hallet K, Joerns SE. Mechanisms of intracranial hemorrhage in infective endocarditis. Stroke; a journal of cerebral circulation 1987;18(6):1048-56. 